\title{
Singlet Pathway to the Ground State of Ultracold Polar Molecules
}

\author{
A. Yang, ${ }^{1}$ S. Botsi, ${ }^{1}$ S. Kumar, ${ }^{1}$ S. B. Pal, ${ }^{1}$ M. Lam, ${ }^{1}$ I, Čepaitė, ${ }^{1}$ A. Laugharn, ${ }^{1}$ and \\ K. Dieckmann ${ }^{1,2}$ \\ ${ }^{1}$ Centre for Quantum Technologies (CQT), 3 Science Drive 2, Singapore 117543, Singapore \\ ${ }^{2}$ Department of Physics, National University of Singapore, 2 Science Drive 3, Singapore 117542, \\ Singapore \\ E-mail: phydk@nus.edu.sg
}

\begin{abstract} association. With the only contributing singlet state to the molecular state being fully stretched and with control over the lasers polarization we address a sole hyperfine component of the excited $\mathrm{A}^{1} \Sigma^{+}$potential without resolving its hyperfine structure. This scheme ensures access to only one ground state hyperfine component with sufficient Franck-Condon factors and moderate laser powers for both coupling transitions. Its implementation results in large and balanced Rabi frequencies, a favourable condition for the coherent transfer to the ground state. We perform dark resonance spectroscopy to precisely determine the transition frequencies of the states involved. The strong dipolar nature of ${ }^{6} \mathrm{Li}^{40} \mathrm{~K}$ is revealed by Stark spectroscopy, as it is necessary for the study of dipolar interactions in an optical lattice.
\end{abstract}

We demonstrate a two-photon pathway to the ground state of ${ }^{6} \mathrm{Li}^{40} \mathrm{~K}$ molecules that involves only singlet-to-singlet optical transitions. We start from a molecular state which contains a significant admixture from the singlet ground state potential by selecting the Feshbach resonance for molecule

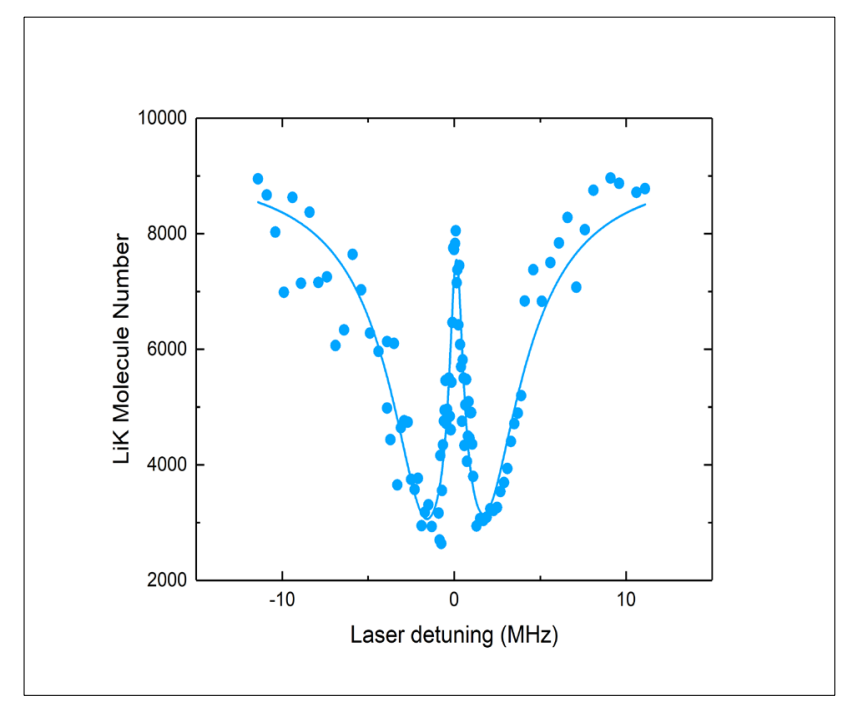

Fig. 1: Two-photon dark resonance spectroscopy of ${ }^{6} \mathrm{Li}^{40} \mathrm{~K}$ molecules. The narrow peak indicates the existence of the dark state.

Keywords: Ultracold Molecules, Molecular Spectroscopy, Feshbach Resonance, Electromagnetically Induced Transparency. 